\title{
Paper Persistence and Computer-based Workarounds with the Electronic Health Record in Primary Care
}

\author{
Jason J. Saleem ${ }^{1-4}$, Mindy Flanagan ${ }^{3}$, Laura G. Militello ${ }^{5}$, Nicole Arbuckle ${ }^{6}$, Alissa L. Russ ${ }^{1-3,7}$, \\ A. Lucile Burgo-Black ${ }^{8,9}$, and Bradley N. Doebbeling ${ }^{1-3,10}$ \\ ${ }^{1}$ VA HSR\&D Center on Implementing Evidence-Based Practice, Roudebush VAMC, Indianapolis, \\ IN; ${ }^{2}$ Regenstrief Institute, Inc., Indianapolis, IN; ${ }^{3}$ Indiana University (IU) Center for Health Services \\ \& Outcomes Research, Indianapolis, IN; ${ }^{4}$ Department of Electrical \& Computer Engineering, IUPUI, \\ Indianapolis, IN; ${ }^{5}$ Applied Decision Science, Kettering, $\mathrm{OH} ;{ }^{6}$ University of Dayton Research Institute, \\ Dayton, $\mathrm{OH} ;{ }^{7}$ Department of Pharmacy Practice, Purdue University College of Pharmacy, West \\ Lafayette, IN; ${ }^{8}$ VA Connecticut Healthcare System; ${ }^{9}$ Yale University School of Medicine, New \\ Haven, CT; ${ }^{10}$ Department of Medicine, IU School of Medicine, Indianapolis, IN.
}

With the United States national goal and incentive program to transition from paper to electronic health records (EHRs), healthcare organizations are increasingly implementing EHRs and other related health information technology (IT). However, in institutions which have long adopted these computerized systems, such as the Veterans Health Administration, healthcare workers continue to rely on paper to complete their work. Furthermore, insufficient EHR design also results in computer-based workarounds. Using direct observation with opportunistic interviewing, we investigated the use of paper- and computerbased workarounds to the EHR with a multi-site study of 54 healthcare workers, including primary care providers, nurses, and other healthcare staff. Our analysis revealed several paper- and computer-based workarounds to the VA's EHR. These workarounds, including clinician-designed information tools, provide evidence for how to enhance the design of the EHR to better support the needs of clinicians.

\section{INTRODUCTION}

The potential benefits for healthcare systems using electronic health records (EHRs) and computerized clinical decision support (CDS) systems are compelling. EHRs provide improved legibility of patient data; simultaneous, remote access; and integration with other information sources (Powsner, Wyatt, \& Wright, 1998). Computer-based CDS, including clinical reminder systems, may increase adherence to preventive care guidelines (Hunt, Haynes, Hanna, \& Smith, 1998). While some of the potential benefits of an EHR and CDS are well-known, they do not produce completely "paperless" processes. Previous research described the phenomenon of "paper-persistence" for medical ordering processes where computerized provider order entry was implemented (Ash et al., 2007; Campbell, Sittig, Ash, Guappone, \& Dykstra, 2006; Dykstra et al., 2009), and identified underlying reasons for the persistence of paper with the EHR in general (Saleem et al., 2009b). Although some of these paper-based workarounds are problematic, others may illustrate efficiencies and other advantages of paper over corresponding electronic workflows, as well as disclose the potential design limitations of computerized systems (Sellen \& Harper, 1997).

Our objectives were two fold. First, we applied the work previously performed on paper persistence in a secondary analysis of data collected from a multi-site study in VA primary care. Second, we extended the work to include computer-based workarounds as well. The identification of paper- and computer-based workarounds to the EHR and CDS systems help us understand how to enhance the design of these systems to better support the needs of clinicians. They also reveal clinician-designed artifacts and alternative workflows that need to be supported and well-coordinated with the existing health information technology (IT).

\section{METHODS}

\section{Study Sites and Participant Characteristics}

Two large, tertiary care Veterans Affairs Medical Center (VAMC) sites were selected based on having a strong medical informatics research presence, strong clinical performance, and a national geographic distribution (east and south). At each site, qualitative data was collected in multiple primary care clinics. For this IRB-approved study, we observed a total of 54 healthcare workers across the two sites. Table 1 shows the participants for each study site, including their clinical roles. VA's EHR, the Computerized Patient Record System (CPRS), is an integrated program with multiple software packages designed to allow providers to order medications, laboratory tests, consultations, as well as document actions and view test results. CPRS includes some CDS; primarily computerized clinical reminders for primary care. CPRS and the tools that comprise it, such as the clinical reminders, were designed to be used by all clinic staff. Thus, we included a wide range of clinical roles in the study.

\section{Field Study Methods}

The researchers conducted direct observation, with opportunistic interviews, of EHR and related CDS use to 
identify putative best practices and barriers to effective use of health IT. For the primary study, there was a particular interest in CDS for colorectal cancer screening (Saleem et al., 2009a). However, observations were recorded for all aspects of the clinician's interaction with the EHR. This enabled us to perform a secondary analysis of the data for workarounds to the health IT, which this paper reports.

TABLE 1: Participant breakdown for the study sites including clinical role

\begin{tabular}{lll}
\hline & \multicolumn{2}{c}{ Study Sites } \\
\cline { 2 - 3 } Role & VAMC 1 (east US) & VAMC 2 (south US) \\
\hline PCP & 6 & 11 \\
$\quad$ Physician & 2 & 2 \\
PA & 1 & 6 \\
NP & 2 & 14 \\
RN/LPN & 8 & 1 \\
HT/MA & 0 & 1 \\
CAC & $\mathbf{1 9}$ & $\mathbf{3 5}$ \\
Total &
\end{tabular}

Note. VAMC $=$ Veterans Affairs Medical Center; $\mathrm{PCP}=$ primary care provider; $\mathrm{PA}=$ physician assistant; $\mathrm{NP}=$ nurse practitioner; $\mathrm{RN}=$ registered nurse; $\mathrm{LPN}=$ licensed practical nurse; $\mathrm{HT}=$ health tech; $\mathrm{MA}=$ medical assistant; $\mathrm{CAC}=$ clinical application coordinator.

Researchers used direct observation to understand the range of ways in which providers interact with and use the EHR and related CDS tools in real time. Direct observation allowed researchers to gather data on the context and process surrounding EHR and CDS use. During observations, two to three observers experienced in ethnographic observation separately shadowed providers as they interacted with the EHR and CDS tools during an actual work shift. There were two observers (JS and LM) for data collected at Site 1. An additional observer (NA) joined the data collection team at Site 2. Also, we observed in four primary care clinics at Site 2; there were only two primary care clinics at Site 1 . The additional observer and additional clinics at Site 2 allowed for the inclusion of several more participants at Site 2 than Site 1 (see Table 1). Observations were recorded via handwritten notes on a structured observation form during participant interaction with the EHR, capturing observable activities and verbalizations.

To better understand the observational data, observers also conducted opportunistic interviews of providers on their use of the EHR in the outpatient clinics. Interviews were conducted so as not to disrupt the natural workflow of the providers. Interview questions covered why providers took certain actions as well as opinions and feedback about barriers to the use of the EHR and CDS. Opportunistic feedback was recorded in the structured observational form. The feedback supplemented and aided understanding of corresponding observations.

\section{Data Collection}

Before each site visit, a local contact person was identified and served as the liaison during data collection. This person introduced the observers and scheduled the observations in outpatient clinics. For each site, investigators conducted observations during two full days in at least two different outpatient clinics. Healthcare workers included in the observations read and signed an informed consent if they chose to participate in the study. Handwritten observations were typed after each site visit, and a scheme was applied to permit tracking of observer, site, clinic, and day.

\section{Data Analysis}

Two authors performed the qualitative analysis (JS and MF) by first independently reviewing the entire body of observation data from the two sites and extracting all observations that seemed relevant to paper persistence and workarounds (i.e., the topic of the secondary analysis). The two researchers then met to reconcile the final extracted data set from which to code (60 of 673 total observations).

Differences in the independently extracted data set were resolved by consensus. Using this subset of data, the two coders independently coded the 60 observations in a two-stage fashion. First, each observation was coded as a paper-based or computer-based workaround. Then, we coded each observation using a 'top-down' application of 11 reasons for the persistent use of paper with health IT found in previous research (Saleem et al., 2009b). This coding scheme was found to be applicable to the computer-based workarounds as well. For observations where none of the 11 established codes seemed relevant, a new code was established to characterize the workaround. All differences in the independently coded observations were resolved by consensus through a series of three additional meetings. Every difference in coding for both coding schemes was discussed by the two researchers until consensus was reached.

\section{RESULTS}

We found examples that fell within 7 of the 11 categories for paper-based workarounds to the EHR reported in previous research (Table 2). Since these categories represent the underlying reason for a workaround, they were also applicable to the computer-based workarounds. One additional category emerged for the computer-based workarounds: 'No correct path' (e.g., there was no desired option in a computer dialog box, precipitating a workaround). Descriptions of each of the categories are as follows:

- Efficiency: Using a workflow process that improves actual or perceived efficiency.

- Awareness: Recognize new / important information: notify, alert, trigger; adjusting "signal to noise" ratio.

- Memory: Reminder about "old" or existing information. 
- Task specificity: Need specificity or ability to customize to patient, provider, department, etc; some signal / noise issues.

- Data organization: Data layout issues; need to view existing data differently.

- Longitudinal data processes: Task requires processing multiple data points across time.

- Task complexity: Complexity of task dictates workflow issues or functionality issues.

- No correct path: Desired actions not possible within the options of the computer interface.

The four categories from Saleem et al., 2009b not apparent in this data set were: knowledge/skill/ease of use, sensorimotor preferences, trust, and security.

TABLE 2: Paper-based workarounds (PBW) and computerbased workarounds (CBW) by category across participant roles

\begin{tabular}{lll}
\hline Category & \multicolumn{1}{c}{$\begin{array}{l}\text { PBW } \\
\text { No. of Subjects } \\
\text { (role) }\end{array}$} & $\begin{array}{l}\text { CBW } \\
\text { No. of Subjects } \\
\text { (role) }\end{array}$ \\
\hline Efficiency & 5 (Phys, 4 HT) & $5(3$ Phys, PA, NP) \\
Awareness & 5 (Phys, 2 RN, 2 HT) & 1 (NP) \\
Memory & $5(\mathrm{PA}, 3 \mathrm{RN}, \mathrm{HT})$ & 1 (NP) \\
Task specificity & $2(\mathrm{PA}, \mathrm{HT})$ & 0 \\
$\begin{array}{l}\text { Data organization } \\
\text { Longitudinal data }\end{array}$ & 1 (RN) & 2 (Phys, NP) \\
\multicolumn{1}{c}{ processes } & 1 (HT) & 0 \\
Task complexity & 1 (Phys) & 0 \\
$*$ No correct path & 0 & $4(2$ Phys, PA, NP) \\
\hline
\end{tabular}

Note. 'No. of Subjects' means the number of subjects with observations coded with the given category. The asterisk denotes a new category not part of the original coding from Saleem et al. (2009b).

\section{DISCUSSION}

This study revealed several paper- and computer-based workarounds to the VA's EHR used by a variety of primary care providers, nurses, and HT/MAs. Many of these alternative workflows and tools genuinely supported the clinicians in their work in important ways where the EHR could not. However, circumventing EHRs via paper or electronic tools introduces the potential for gaps in documentation as well as the unintentional propagation of errors. The use of workarounds to improve efficiency, or perceived efficiency, was the most prevalent reason for both paper persistence and computer-based workarounds (Table 2). Workarounds based on memory and awareness were also common. Thus, we focus the majority of the discussion on these categories.

\section{Every Minute Counts}

Clinicians valued strategies for saving time and becoming more efficient in their work. For several of the cases of paper persistence related to efficiency, a 'role division' strategy was present. For example, many of the HTs at Site 1 worked together to record patient vitals and complete screening clinical reminders. One of the HTs was informally designated as the computer entry person while the other HTs took the patients' vitals (weight, blood pressure, etc.) and simultaneously asked the screening questions to complete the computerized reminders (e.g., 'Did you ever smoke or use chew or snuff?'). The designated computer entry HT entered the information into the EHR in real time as the others verbally relayed the information. If he/she was not present (e.g., on a break), the other HTs would handwrite the vitals and screening reminder responses on paper for the designated 'computer HT' to enter later. This type of informal role assignment was perceived to improve overall efficiency of the patient intake process.

Several more cases of paper persistence coded as efficiency related to a 'consolidation of tasks' strategy, often done in a proactive manner. For example, one RN explained that at the start of the day, she prints a form for each patient she will see that day. On the form, she writes down the clinical reminders that are due, any blood work needed, and so forth. She keeps the printed forms in her pocket and uses them to record each patient's vitals. When she has an opportunity to return to her workstation, she enters all of the information into the computer. This type of proactive strategy by nurses has also been described in parallel research in non-VA settings (Patterson, Ebright, \& Saleem, 2011). In addition to efficiency, this case was also coded as 'memory' (use of the paper form as a memory aid).

Computer-based workarounds coded as efficiency related to using alternative and unexpected computer workflows to complete a task as well as copying and pasting text from the patient's previous progress note to the new one. For the former case, an example included a PA completing a computerized Gastroenterology (GI) consult request to order a colonoscopy instead of completing the appropriate colorectal cancer (CRC) screening clinical reminder and ordering the procedure through the reminder dialog box. While the strategy of entering a consult was more efficient for the PA, it defeated the purpose of the enhanced CRC clinical reminders, which include a series of reminders for screening, follow-up, and surveillance. Without completing the appropriate CRC reminder, the patient's CRC care is more difficult to track through the EHR. Copying and pasting text from a previous progress note to a new one was another computer-based workaround done for efficiency reasons. The risk with this strategy, however, includes potential propagation of errors from the previous note or even mistakenly copying text into a different patient's record (Hammond, Helbig, Benson, \& Brathwaite-Sketoe, 2003). 


\section{Temporary Cognitive Cues}

Use of paper as temporary cognitive cues in conjunction with the EHR for reasons based on memory or awareness were coded across several participants (Table 2). Use of paper 'scraps' as memory aids were documented especially with the nursing staff. This finding is consistent with other research specific to nursing (Hardey, Payne, \& Coleman, 2000). Examples of this practice in our data included recording patient vitals on paper 'scraps' for later computer entry and using EHR printouts as reminders to follow-up on certain items. A computer-based example of a memory aid involved a NP recording a recommendation in the Active Problems summary list on the coversheet of the EHR that the patient needed another colonoscopy in 3 years. She added this recommendation to make sure any clinician would see it upon accessing the patient's record instead of the recommendation being "buried" in a progress note. This was a workaround in the sense that it was not a true problem list item like diagnosed 'hypertension' or 'diabetes'.

Cases involving awareness were distinguished from memory when they involved new information. A common one was for a HT, MA, or RN to alert the PCP with handwritten notation on an EHR printout. For example, an RN informed a patient he was due for a fecal occult blood test (FOBT). The patient refused the FOBT and she wrote the refusal on the paper form for the PCP to see. She also checked 'Patient refused FOBT cards' in the colorectal cancer screening computerized reminder. This result is visible to the PCP through the EHR. However, the RN and others who used handwritten alerts did not believe the PCP would necessarily notice this result in the EHR.

\section{No Correct Path}

Although the coding scheme for the underlying reasons for paper persistence also applied well to the computer-based workarounds, a single new code emerged, unique to the computer-based cases: 'No correct path'. These cases involved a clinician confronted with no options available on the computer interface to conduct his/her desired action. For example, a physician was completing a tobacco screening computerized clinical reminder. He noted that he was going to prescribe Chantix (varenicline), which was not one of the choices in the dialog box. He clicked on 'patient will get nicotine treatment outside the VA', even though this was inaccurate, simply to get the reminder to "go away". Another example involved a physician instructing a patient to take half a pill. The physician said, "We tell them to split that pill but the CPRS med list will only show the full pill and then there's a mismatch [in terms of timing when the EHR indicates the medication needs to be renewed]." These cases demonstrate how clinicians sometimes need to work around a rigid EHR design.

\section{User-Designed Information Tools}

Gurses et al. describe the concept of "user-designed information tools"; these are sophisticated paper workarounds developed by clinicians based on the inability of the existing health IT to meet the their needs (Gurses, Xiao, \& Hu, 2009). Two of the paper artifacts categorized as 'task specificity' in the results are examples of user-designed information tools. The first was referred to by a HT as a "Clinical Reminder Sweep Notebook" (Site 2). This clinician-designed artifact was created in response to the clinic performing poorly against national quality indicators, which are tied directly to the completion of the computerized clinical reminders. The intent was to develop a tool that would allow them to look across patients to identify everyone who was due for a specific test or intervention. For example, the clinical reminder for colorectal cancer screening was tracked in the notebook. For each patient, the HT recorded in the notebook their phone number and each time the patient was called and reminded to return their FOBT cards. For any clinical reminders missed for a patient, the HT prints a copy of the reminder and shows it to the nurse and provider so they could follow-up. In many cases, this pro-action facilitated by the notebook artifact enabled the clinic to meet the deadline before the reminder was recorded as being late. This type of tracking across a panel of patients (i.e., task specificity) was not possible with the EHR.

Another clinician-designed paper artifact, coded as both 'task specificity' and 'longitudinal data processes', was a spreadsheet designed to track the last date of a colonoscopy for patients who received colonoscopies outside of the VA system, the location of the procedure, and the results. This type of unofficial, shadow documentation was shared between at least one physician and one PA at Site 1 and allowed for specifying a certain group of patients and tracking multiple data points across time. Neither of these affordances was provided by the EHR. These types of clinician-design information tools provide evidence for the information needs of the clinicians, not sufficiently supported by the EHR, for improving EHR design.

\section{Applicability to other Healthcare Organizations}

The results from this paper are applicable to other healthcare organizations that use an EHR or are planning to implement one since the paper persistence codes can be applied outside the VA. The underlying reasons for paper persistence in the presence of an EHR are universal: clinicians will create their own paper processes when it is more efficient, when it serves as an important cognitive memory aid, when it can better alert them than the computer system, and so forth. Therefore, knowing why the VA clinicians have developed paper- and computer-based workarounds to improve their work efficiency, for example, will inform others who intend to implement a system or help them understand why paper persists in their organization and why clinicians are using the EHR in ways it was not originally intended or designed to be used. 


\section{Conclusion}

We applied a coding scheme to examine the reasons why paper persists with the EHR in a secondary analysis of data collected from a multi-site study in VA primary care. Cases for seven of the original 11 categories were present in this analysis. These findings help substantiate the original study on the persistence of paper with the EHR (Saleem et al., 2009b), which was performed at a different VA study site than the two sites included in this study. See also Saleem et al. (2011) for the same codes applied to computerized consultations. During the coding, we allowed for the emergence of new categories not present in the original 11; however, there were no cases that qualified for a new category of paper persistence. We also successfully applied the same coding to computer-based workarounds as the underlying reasons that drive the paperbased workarounds were applicable. An additional code, 'no correct path', was needed to describe some of the computerbased workarounds unique to the rigidity of computer vs. paper-based systems.

The clinician-designed information tool examples demonstrated how clinicians designed their own "shadow documentation"(Wears, 2008; Wears \& Perry, 2008) to serve their needs related to the specificity of the clinical task (i.e., customizing information views for a panel of patients). The EHR tends to be designed as a single patient-centric tool. Future EHR design should account for the need to customize data across a panel of related patients. Specific cases of paperand computer-based workarounds documented in this study provide an opportunity to innovate EHR design to better support clinical work in primary care. Without the corresponding design innovations, the EHR must at least be coordinated with the information tools and workarounds to prevent potential gaps in documentation and errors resulting from the lack of integration with informal information sources.

\section{ACKNOWLEDGEMENTS}

This research was supported under contract number HHSA 290-06-00013-3 from the Agency for Healthcare Research and Quality (AHRQ), U.S. Department of Health and Human Services. Dr. Saleem is supported by a VA HSR\&D Career Development Award (CDA 09-024-1). This work is also supported in part by the VA HSR\&D Center of Excellence on Implementing Evidence-Based Practice (CIEBP), HSR\&D Center grant \#HFP 04-148. The views expressed in this article are those of the authors and do not reflect the official position of AHRQ, U.S. Department of Health and Human Services, or the Department of Veterans Affairs.

\section{REFERENCES}

Ash, J. S., Sittig, D. F., Poon, E. G., Guappone, K., Campbell, E., \& Dykstra, R. H. (2007). The extent and importance of unintended consequences related to computerized provider order entry.

J.Am.Med.Inform.Assoc., 14, 415-423.
Campbell, E. M., Sittig, D. F., Ash, J. S., Guappone, K. P., \& Dykstra, R. H. (2006). Types of unintended consequences related to computerized provider order entry. J.Am.Med.Inform.Assoc., 13, 547-556.

Dykstra, R. H., Ash, J. S., Campbell, E., Sittig, D. F., Guappone, K., Carpenter, J. et al. (2009). Persistent paper: The myth of "going paperless". AMIA.Annu.Symp.Proc., 158-162.

Gurses, A. P., Xiao, Y., \& Hu, P. (2009). User-designed information tools to support communication and care coordination in a trauma hospital. J.Biomed.Inform., 42, 667-677.

Hammond, K. W., Helbig, S. T., Benson, C. C., \& BrathwaiteSketoe, B. M. (2003). Are electronic medical records trustworthy? Observations on copying, pasting and duplication. AMIA.Annu.Symp.Proc., 269-273.

Hardey, M., Payne, S., \& Coleman, P. (2000). 'Scraps': hidden nursing information and its influence on the delivery of care. J.Adv.Nurs., 32, 208-214.

Hunt, D. L., Haynes, R. B., Hanna, S. E., \& Smith, K. (1998). Effects of computer-based clinical decision support systems on physician performance and patient outcomes: a systematic review. JAMA, 280, 13391346.

Patterson, E. S., Ebright, P. R., \& Saleem, J. J. Investigating stacking: How do registered nurses prioritize their activities in real-time? (2011, in press). Int.J.Indust.Ergonomics, Special Issue on Human Factors and Ergonomics in Healthcare Delivery.

Powsner, S. M., Wyatt, J. C., \& Wright, P. (1998). Opportunities for and challenges of computerisation. Lancet, 352, 1617-1622.

Saleem, J. J., Militello, L. G., Arbuckle, N., Flanagan, M., Haggstrom, D. A., Linder, J. A. et al. (2009a). Provider perceptions of colorectal cancer screening clinical decision support at three benchmark institutions. AMIA.Annu.Symp.Proc., 2009, 558-562.

Saleem, J. J., Russ, A. L., Justice, C. F., Hagg, H., Ebright, P. R., Woodbridge, P. A. et al. (2009b). Exploring the persistence of paper with the electronic health record. Int.J.Med.Inform., 78, 618-628.

Saleem, J. J., Russ, A. L., Neddo, A., Blades, P. T., Doebbeling, B. N., \& Foresman, B. H. (2011). Paper persistence, workarounds, and communication breakdowns in computerized consultation management. Int.J.Med.Inform., 80, 466-479.

Sellen, A. \& Harper, R. (1997). Paper as an analytic resource for the design of new technologies. Proceedings of the SIGCHI Conference on Human Factors in Computing Systems, 319-326.

Wears, R. L. (2008). The chart is dead - Long live the chart. Ann Emerg Med., 52, 390-391.

Wears, R. L. \& Perry, S. J. (2008). Semper Gumby sub rosa: Adaptability in a healthcare setting. Proc Human Factors and Ergonomics Society's 52nd Annual Meeting, 319-322. 